\section{Children's Cardiology Up-to-Date Online Resources for Education (CUORE) Project: Remote Education for Training in Pediatric Critical Care Medicine}

To the Editor-The Children's Cardiology Up-to-Date Online Resources for Education (CUORE) project was a pilot collaborative initiative between clinicians in the cardiac intensive care unit (ICU) at Children's Hospital Boston and the pediatric ICU of the Catholic University Hospital in Santiago, Chile. The primary aim of this pilot study was to evaluate the feasibility of a remote education program to share information between Children's Hospital Boston and Catholic University Hospital in Santiago as an opportunity to improve standards of care in a pediatric ICU with more limited resources. We chose the prevention of healthcare-acquired infection in the pediatric critical care setting as our initial program to enhance quality outcomes, with the primary hypothesis that the application of a multifaceted educational intervention aimed at increasing provider awareness of evidence-based infection control practices would lower the incidence of central line-associated bloodstream infections (CLABSIs) in the "remote" pediatric ICU in Chile.

We employed standard video-teleconferencing equipment and connected directly using an Internet protocol, since highbandwidth Internet access was available to both participating ICUs. The intervention consisted of 4 online conferences each month during a 5-month period (July-November 2009) and a fifth conference in May 2010. We discussed a quality improvement model based on 5 main interventions: (1) discussion of current Centers for Disease Control and Prevention recommendations, (2) improvement of compliance with hand hygiene (education of staff about hand hygiene and creation of new hand hygiene signs), (3) education to decrease the incidence of CLABSIs (eg, discussion of central venous catheter insertion and use of maximal sterile barriers), (4) creation of a central catheter procedure cart and kit, and (5) empowerment of nurses to stop any nonemergency procedures if guidelines for central venous catheter insertion were not being followed. ${ }^{1,2}$

After each presentation, there was interactive discussion among participants regarding each topic. All documents and PowerPoint (Microsoft) slides were translated into Spanish, and all presentations and discussions were conducted in Spanish by a native Spanish speaker. Additional conferences in Santiago among Chilean clinicians were also held each week. These conferences aimed at reinforcing evidence-based practices discussed during the monthly online conferences. A total of 20 weekly conferences were held. Each weekly conference reached a mean of 24 additional pediatric ICU health- care professionals. Further questions and explanations that rose from group discussions in Santiago were addressed by e-mail. A postintervention survey of participants at Catholic University Hospital in Santiago was conducted regarding comments on conference content and to generate ideas for future topics and improvement. The mean number of CLABSIs per 1,000 catheter-days showed no significant difference between the preintervention and postintervention periods. The response rate to the questionnaire was $75 \%$; of the individuals who answered, $100 \%$ agreed that the topics discussed were relevant to their practice and that the length of conferences was appropriate, $77 \%$ reported that the material was presented at the right level, and 55\% reported that they would maintain the format of the initiative as it was. According to responses to open-text questions, future conferences should focus on strategies aimed at the reduction of ventilator-associated pneumonia, urinary tract infection, and surgical wound infection, as well as the prevention of healthcare-acquired infections in immunocompromised patients.

Digitized health care now allows the outsourcing of a variety of medical services, from clinical diagnostics to direct care. ${ }^{3}$ The prospective benefits of these instruments are particularly significant in countries where specialists are few and access to up-to-date medical knowledge is limited. Although CUORE demonstrated the feasibility of an interactive collaboration between clinicians at Children's Hospital Boston and Catholic University Hospital in Santiago, we were unable to show any reduction in CLABSI rates from the preintervention to the postintervention periods in Santiago. There are a number of likely reasons for this lack of effect, which also demonstrate the limitations of this study. The risk for CLABSI may have been different between the critical care units themselves, since patient demographics, care, and interventions provided were neither identical nor standardized during the pilot study. The preintervention mean CLABSI rates reported for the pediatric ICU of the Catholic University Hospital in Santiago were low compared with the international National Healthcare Safety Network benchmark (5.3 BSIs per 1,000 device-days, in 2006). ${ }^{4}$ An established program for infection control was already in place at Catholic University Hospital in Santiago before the CUORE program started, and some of the interventions noted above had already been implemented. Given the limited time frame for the study and relatively small sample size, it would be difficult to expect a significant difference in rates between the preintervention and postintervention periods. The literature regarding single center efforts to reduce CLABSI rates has consistently demonstrated a lag effect between the initial intervention efforts and a significant decline in CLABSI rates. ${ }^{2}$ We had only 1 postintervention period of 5 months (through May 2010) to report, which is too short to demonstrate a sustained reduction in infection rate. We did not assess preintervention knowledge 
deficits regarding CLABSI prevention, nor were we able to obtain postintervention data on compliance with hand hygiene and central venous catheter access and maintenance bundles.

Further work is planned for ongoing collaboration and tracking of infection rates over time. Despite these limitations, the CUORE pilot project demonstrated that real-time interactive conferences for healthcare providers placed in remote pediatric ICUs are feasible and yield a high satisfaction among participants. Future efforts should concentrate on sustaining online training programs for a longer time, eventually involving multiple centers at the same time. We believe this model would also be appropriate for critical care topics other than CLABSI rate reduction. In addition to continuing the CUORE program, we are aiming at developing further online resources (eg, a CUORE Web site containing lecture material).

\section{ACKNOWLEDGMENTS}

A special thank-you to Esther Baena, PhD, for her invaluable contribution and never-ending support.

Potential conflicts of interest. All authors report no conflicts of interest relevant to this article.

Angelo Polito, MD, MPH; ${ }^{1,2}$ Cristian Claveria, $\mathrm{MD}^{3}$ Debra Forbes Morrow, RN; ${ }^{1}$ Daniel Springmuller, $\mathbf{M D}^{1,3}$ Dionne Graham, $\mathrm{PhD} ;^{4}$ Vamsi Yarlagadda, $\mathrm{MD}{ }^{1}$ John M. Costello, MD, MPH; Ravi R. Thiagarajan, MBBS, MPH; Peter C. Laussen, MBBS

Affiliations: 1. Division of Cardiac Intensive Care, Department of Cardiology, Children's Hospital Boston, Boston, Massachusetts; 2. Department of Cardiology, Bambino Gesù Children's Hospital, Rome, Italy; 3. Department of Pediatrics, Universidad Catolica de Chile, Santiago, Chile; 4. Clinical Research Program, Children's Hospital Boston, Boston, Massachusetts.

Address correspondence to Angelo Polito, MD, MPH, Dipartimento Medico Chirurgico di Cardiologia Pediatrica, Opsedale Pediatrico Bambino Gesu', Piazza S. Onofrio 4, 00165, Rome, Italy (angpolito@hotmail.com). Infect Control Hosp Epidemiol 2011;32(6):628-629

(C) 2011 by The Society for Healthcare Epidemiology of America. All rights reserved. 0899-823X/2011/3206-0017\$15.00. DOI: 10.1086/660201

\section{REFERENCES}

1. O'Grady NP, Alexander M, Dellinger EP, et al. Guidelines for the prevention of intravascular catheter-related infections. The Hospital Infection Control Practices Advisory Committee, Center for Disease Control and Prevention. Pediatrics 2002;110:e51.

2. Costello JM, Morrow DF, Graham DA, Potter-Bynoe G, Sandora TJ, Laussen PC. Systematic intervention to reduce central lineassociated bloodstream infection rates in a pediatric cardiac intensive care unit. Pediatrics 2008;121:915-923.

3. Singh SN, Wachter RM. Perspectives on medical outsourcing and telemedicine: rough edges in a flat world? $N$ Engl J Med 2008; 358:1622-1627.

4. Edwards JR, Peterson KD, Andrus ML, et al. National Healthcare
Safety Network (NHSN) report, data summary for 2006, issued June 2007. Am J Infect Control 2007;35:290-301.

\section{Community and Nursing Home Residents with Carbapenemase-Producing Klebsiella pneumoniae Infection}

To the Editor-Healthcare-associated carbapenemase-producing Klebsiella pneumoniae (CPKP) infections have a high mortality. ${ }^{1-3}$ In March 2009, the first patient with a CPKP infection identified at the time of presentation to our hospital system was a local nursing home resident. Then another resident with a CPKP infection presented to our hospital system from the same nursing home. A point-prevalence survey was performed using rectal swab samples to assess patients at that nursing home unit. One additional patient from 41 patients screened was identified as a carrier of CPKP. Other cases of CPKP infection were then noted at hospital admission among patients from other nursing homes and from the community. Because there are currently few data on the epidemiology of CPKP infection in patients presenting to US hospitals and clinics, we studied such patients who presented to our hospital system, and we screened nursing home residents at hospital admission to detect asymptomatic CPKP colonization.

We reviewed patients for whom CPKP was grown from clinical cultures in 2009 using the methodology described below at hospital admission to Rhode Island Hospital, Miriam Hospital, or Newport Hospital; during outpatient clinic or emergency department visits at these hospitals; or at a nursing home if the patient's medical care was delivered in our hospital system during 2009. Patients without clinical evidence of infection and admitted patients with samples for cultures that were collected more than 24 hours after hospitalization were excluded. Patient characteristics and risk factors were assessed by medical record review and interviews with primary care physicians and nursing home staff. Functional status was defined as poor if patients were bed-bound or required maximal assistance for ambulation.

During January and February 2010, we screened 100 nursing home patients within 24 hours after admission to the above-noted hospitals using rectal swabs to detect asymptomatic CPKP carriage. Initial CPKP identification was performed using the method recommended by the Centers for Disease Control and Prevention. ${ }^{4}$ Swabs were used to inoculate $5 \mathrm{~mL}$ of Trypticase soy broth (BD) onto a $10-\mu \mathrm{g}$ meropenem disk. After overnight incubation, the broth was vortexed, and $100 \mu \mathrm{L}$ was subcultured onto MacConkey agar, incubated for 48 hours, and examined for lactose-fermenting colonies. The modified Hodge test was performed to confirm carbapenemase resistance. ${ }^{5}$ Our Institutional Review Board approved this project. 